\section{Difficult Defecation Caused by a Huge Rectal Leiomyoma}

Sir,

Although leiomyomas can develop anywhere in the gastrointestinal tract, the stomach and small intestine are more common locations. ${ }^{1}$ Leiomyomas of the rectum are quite rare, which represent only $3 \%$ of all gastrointestinal leiomyomas, and 1 in 2,000 of all rectal tumours. ${ }^{2}$ Most rectal leiomyomas are small and asymptomatic. However, as they grow larger, symptoms such as pain and rectal bleeding can occur.

Rectal leiomyomas can be detected as submucosal tumours by diagnosticmodalitiessuchasendoscopy, endoscopicultrasonography (EUS), and radiological examination. A definitive diagnosis necessitates histological and immunohistochemical examinations. Histological characteristics of leiomyomas are fascicles of spindle cells. Immunohistochemically, leiomyomas are positive for smooth muscle actin and desmin, and negative for CD117 (c-kit). ${ }^{3}$

Rectal leiomyomas should be surgically removed, as they are insensitive to both chemotherapy and radiotherapy. Rectal leiomyomas that present as small intraluminal lesions can be resected by endoscopic surgery. ${ }^{4}$ Common surgical options for extramural or large-sized rectal leiomyomas include transanal resection, low anterior resection, and abdominoperineal resection.

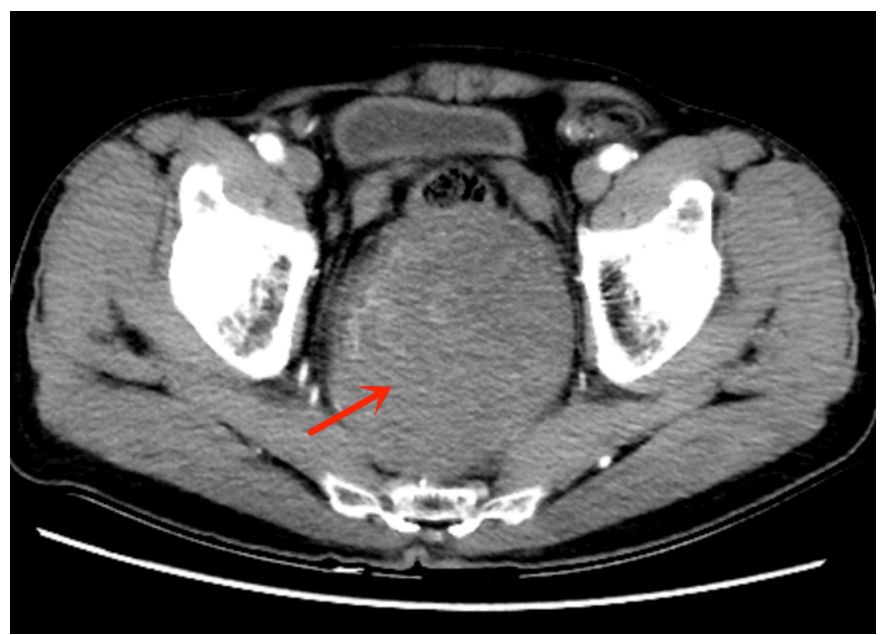

Figure 1: Computed tomography scan demonstrating a giant tumour within the pelvis located dorsal to the rectum (arrow).

A 71-year man presented in outpatient clinic with complaints of difficulty in defecation with small and finger-sized stools for six months. The patient had no history of abdominal pain, diarrhoea or haematochezia. Abdominal examination showed soft abdomen with no masses. Rectal examination was rather limited by a huge firm mass obliterating the rectal lumen. Computed tomography examination revealed a mass within the pelvis, located dorsal to the rectum (Figure 1). EUS showed the tumour to be originating from the third layer of the rectum.

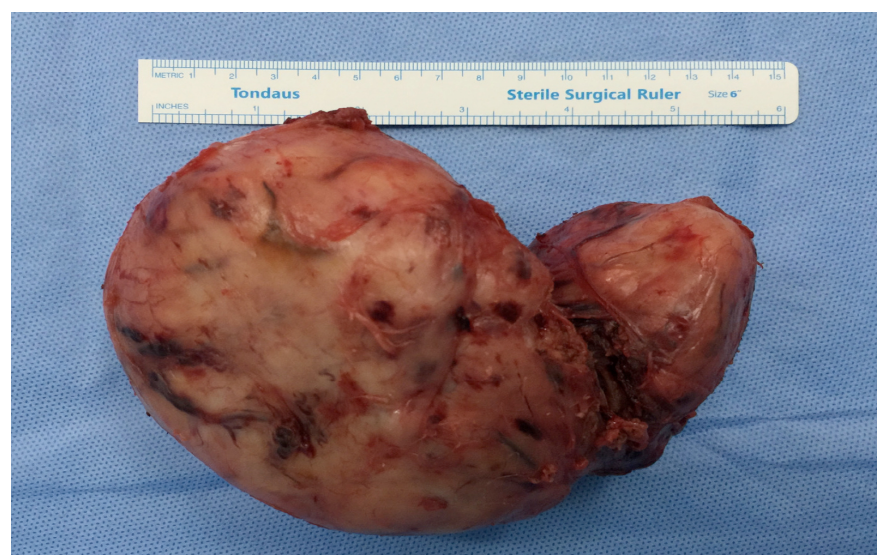

Figure 2: Photograph of the gross specimen.

The patient underwent surgery. Initially, low anterior approach was planned. However, the tumour was so huge that its lower margin could barely be seen. Hence, an extra trans-sacral incision was performed to expose the lower margin of the mass. Subsequently, the tumour measuring $15 \times 10 \times 8 \mathrm{~cm}$ was successfully removed with its capsule intact (Figure 2 ). Considering the possibility of faecal fistula, due to weakness in the posterior wall of the rectum, a diverting stoma was created. The recovery was uneventful. Histopathology and immunohistochemistry identified the mass as a leiomyoma. The diverting stoma was closed three months later after the surgery. The patient was disease-free during six month follow-up.

A giant extramural rectal leiomyoma can be resected by a combination of low anterior approach and trans-sacral approach. This procedure should be recommended as a possible option in the treatment of similar rectal tumours.

\section{CONFLICT OF INTEREST:}

The authors declared no conflict of interest.

\section{AUTHORS' CONTRIBUTION:}

FC, YL: Surgery, paperwriting, literature review.

SZ: Literature review, manuscript revision.

\section{REFERENCES}

1. Zurac S, Tudose I, Micu G, Bastian A, Gamada E, Stăniceanu F, et al. Rectal leiomyoma--report of two cases originating in muscularis mucosae. Rom J Intern Med 2009; 47:97-100.

2. Sunkara T, Then EO, Culliford A, Gaduputi V. Rectal leiomyoma, a rare entity. Clin Pract 2018; 8(2):1053. doi: 10.4081/cp.2018.1053.

3. Kuhlgatz J, Sander B, Golas MM, Gunawan B, Schulze T, Schulten $\mathrm{HJ}$, et al. Differential diagnosis of gastrointestinal leiomyoma versus gastrointestinal stromal tumor. Int J Colorectal Dis 2006; 21(1):84-8. doi: 10.1007/s00384004-0730-1.

4. Lee SH, Huh GY, Cheong YS. A case of endoscopic resection of a colonic semipedunculated leiomyoma. J Korean Soc 
Coloproctol 2011; 27(4):215-9. doi: 10.3393/jksc.2011. 27.4.215.

Feng Chi, Yuefu Lan and Shenkang Zhou

Department of Gastrointestinal Surgery, Taizhou Hospital, Zhejiang University, Zhejiang, China
Correspondence to: Shenkang Zhou, Department of Gastrointestinal Surgery, Taizhou Hospital, Zhejiang University, Zhejiang, China

E-mail: zhoushenkang@yeah.net

Received: January 14, 2021; Revised: January 28, 2021; Accepted: April 21, 2021

DOI: https://doi.org/10.29271/jcpsp.2022.01.135 